\title{
Efficient room-temperature spin detector based on GaNAs
}

Yuttapoom Puttisong, Irina Buyanova, L Geelhaar, H Riechert, C W Tu and Weimin Chen

\section{Linköping University Post Print}

N.B.: When citing this work, cite the original article.

Original Publication:

Yuttapoom Puttisong, Irina Buyanova, L Geelhaar, H Riechert, C W Tu and Weimin Chen, Efficient room-temperature spin detector based on GaNAs, 2012, Journal of Applied Physics, (111), 7, 07C303.

http://dx.doi.org/10.1063/1.3671781

Copyright: American Institute of Physics (AIP)

http://www.aip.org/

Postprint available at: Linköping University Electronic Press

http://urn.kb.se/resolve?urn=urn:nbn:se:liu:diva-78283 


\title{
Efficient room-temperature spin detector based on GaNAs
}

\author{
Y. Puttisong, ${ }^{1}$ I. A. Buyanova, ${ }^{1}$ L. Geelhaar, ${ }^{2}$ H. Riechert, ${ }^{2}$ C. W. Tu, ${ }^{3}$ and W. M. Chen ${ }^{1, a)}$ \\ ${ }^{1}$ Department of Physics, Chemistry and Biology, Linköping University, S-581 83 Linköping, Sweden \\ ${ }^{2}$ Paul-Drude-Institut für Festkörpelektronik, 10117 Berlin, Germany \\ ${ }^{3}$ Department of Electrical and Computer Engineering, University of California, La Jolla, CA 92093, USA
}

(Presented 3 November 2011; received 23 September 2011; accepted 20 October 2011; published online 15 February 2012)

\begin{abstract}
Efficient and highly spin-dependent recombination processes are shown to not only turn GaNAs into an efficient spin filter but also to make it an excellent spin detector functional at room temperature (RT). By taking advantage of the defect-engineered spin-filtering effect, the spin detection efficiency is no longer limited by the fast spin relaxation of conduction electrons. This leads to a significant enhancement in the optical polarization of the spin detector, making it possible to reliably detect even very weak electron spin polarization at RT, as demonstrated by a study of spin loss during optical spin injection across a GaAs/GaNAs interface.
\end{abstract}

(C) 2012 American Institute of Physics. [doi:10.1063/1.3671781]

The efficient generation, transport, and detection of spinpolarized electrons are essential to future spintronics. ${ }^{1-5}$ Until now, most earlier studies have been restricted to low temperatures, at which spin effects are usually more pronounced due to the suppression of spin loss. Spin generation and detection at room temperature (RT) remain difficult and unresolved, however, particularly in semiconductor systems that are considered to potentially provide an excellent platform for the integration of electronics, photonics, and magnetics. Relievable RT spin detectors, as a key element in applications of spintronic devices and a means in the fundamental studies of spin injection and spin loss processes, are still lacking, in general. One of the most widely used methods in spin detection is by exploiting the optical polarization of band-to-band (BB) transitions in semiconductors, which directly measures the spin polarization of conduction electrons determined by the well-known selection rules of optical transitions. ${ }^{6}$ Such optical spin detection has been shown to be very successful at low temperatures, but has unfortunately failed to provide sufficient efficiency for reliable spin detection at RT due to the accelerated spin relaxation, as illustrated in Fig. 1(a). In this work, by taking advantage of a recently discovered defect-engineered spin-filtering effect in GaNAs, ${ }^{7}$ we explore the potential of this material for efficient RT spin detection. We shall show that by transferring the spin orientation from conduction band (CB) electrons to electrons bound to spin-filtering defects that have a much longer spin lifetime, the spin polarization of the former is no longer limited by their own fast spin relaxation. This leads to a significant enhancement in spin polarization and in the intensity of the BB transitions, which enables us to detect even very weak electron spin polarization that is often encountered at RT. The effectiveness of such a spin detector is demonstrated by a case study of optical spin injection from a GaAs spin injection layer to an adjacent GaNAs spin detector.

The studied GaAs/GaNAs structures were grown by molecular beam epitaxy at temperatures, $\mathrm{T}_{\mathrm{g}}$, of $390-580{ }^{\circ} \mathrm{C}$ on a

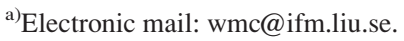

(001)-oriented GaAs substrate. The growth started with a $2500 \AA$ Å-thick GaAs buffer, followed by either a GaNAs epilayer (about $1000 \AA$ in thickness) or 7-period GaAs/GaNAs (200/70 §) multiple quantum-wells, and finally capped by a GaAs layer (200-1000 $\AA$ thick). The $\mathrm{N}$ compositions of the GaNAs epilayers are in the range of 1.3-2.6\%. Optical orientation experiments were performed in a backscattering geometry, with both the excitation and detection beams directed along the growth axis of the samples. A Ti-sapphire laser was employed for optical excitation at wavelengths of 750-980 nm. The resulting photoluminescence (PL) signals were dispersed by a monochromator and detected by a cooled Ge detector. A [1/4]-wave plate, in combination with a linear polarizer, was used for the generation (or analysis) of the circular polarization of the excitation beam (or PL emissions). All experiments were carried out at RT.

First, we briefly describe the principle of efficient spin detection mediated by the defect-engineered spin filtering effect. Once CB electrons of a non-zero spin polarization enter such a spin detector, e.g., generated by electrical injection or by optical spin orientation under circularly polarized excitation, they can dynamically polarize the spin of the first electron bound to a paramagnetic spin-filtering defect via spin-dependent recombination (SDR) toward the same orientation..$^{7-11}$ As a result, the capture of a second electron by the defect can occur only if the captured CB electron has an opposite spin orientation (i.e., minority spin) due to the Pauli exclusion principle. In other words, when the spinfiltering effect is in action, the spin-filtering defects selectively filter out the minority-spin $\mathrm{CB}$ electrons, whereas the majority-spin $\mathrm{CB}$ electrons remain intact due to a spin blockade, as shown in Fig. 1(b). This spin-filtering process drives the defect and CB electrons toward complete spin polarization, when electron capture and recombination via the spinfiltering defects will cease to occur. Such strong spin polarization can be maintained regardless of the spin relaxation of the $\mathrm{CB}$ electrons. This is due to the fact that as soon as a CB electron makes a spin flip it will immediately be captured and depleted from the $\mathrm{CB}$ by the spin-filtering defects, 


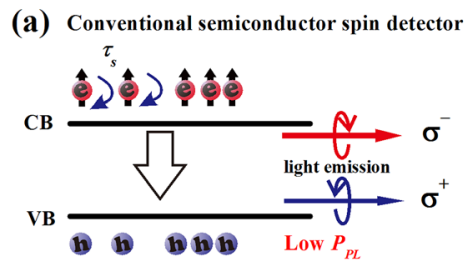

(b) Spin-filtering enabled spin detector
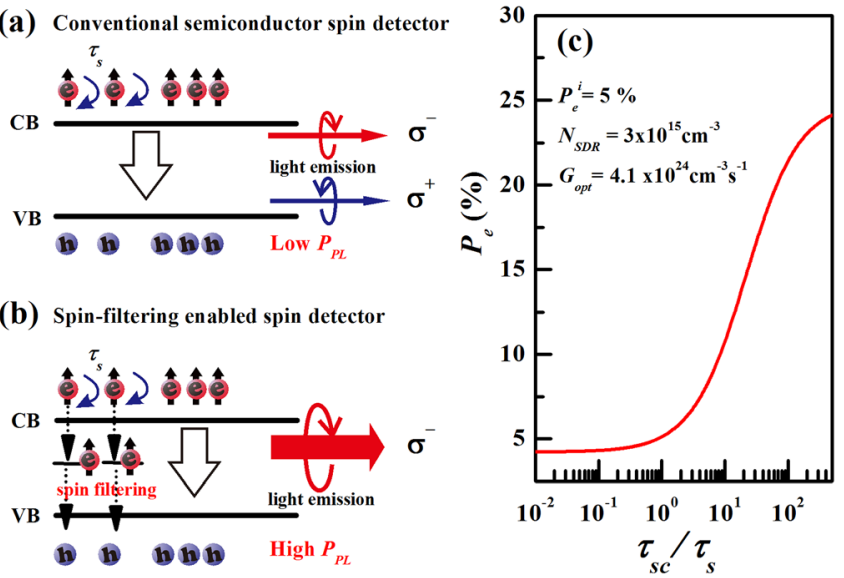

FIG. 1. (Color online) Schematic pictures of (a) a conventional semiconductor spin detector, and (b) a spin-filtering enabled spin detector. (c) The CB electron spin polarization of a spin detector as a function of the ratio between the spin lifetimes of the defect electron $\left(\tau_{s c}\right)$ and the CB electrons $\left(\tau_{s}\right)$, calculated from the coupled rate equations describing the defectengineered spin filtering effect with the specified parameters and $\tau_{s}=150 \mathrm{ps}$ (see Refs. 7-11). The $N_{S D R}$ denotes the total concentration of the spin-filtering defects and $G_{\text {opt }}$ denotes the total generation rate of the $\mathrm{CB}$ electrons by optical excitation. In the simulation, the capture coefficient of the $\mathrm{CB}$ electrons by the defects is assumed to be four times larger than that of the VB holes.

provided that the capture is much faster than the spin flip. This is valid as long as the electrons localized at the defects retain their spin orientation, i.e., possessing a much longer spin lifetime than $\mathrm{CB}$ electrons, which is generally true in semiconductors (especially for deep-level defects such as the spin-filtering defects). The spin detection efficiency is expected to increase with the increasing spin lifetime of the defects, as illustrated in Fig. 1(c) from an analysis by the coupled rate equations. ${ }^{7-11}$ When the initial spin polarization of the $\mathrm{CB}$ electrons is zero, e.g., zero electrical spin injection or under $\sigma^{x}$ excitation, the aforementioned spin blockade should be absent as the defect electrons are not spin polarized. In this case, the defects will freely capture the CB electrons of both spin orientations and recombine them with valence band (VB) holes. This difference in the spin blockade between the cases with and without spin injection (e.g., between $\sigma^{+}$and $\sigma^{x}$ excitation) can lead to a corresponding difference in the PL intensity between these two conditions, i.e., giving rise to the so-called SDR ratio, $I^{\sigma^{+}} / I^{\sigma^{x}}>1$. The efficiency of the spin filtering and spin blockade effect, before reaching a saturation level, should strongly depend on the initial spin polarization, $P_{e}^{i}$, of the injected $\mathrm{CB}$ electrons. Therefore, both the spin polarization and density of the $\mathrm{CB}$ electrons resulting from the spin filtering effect are direct measures of $P_{e}^{i}$. The corresponding two types of spin detector signals are thus the optical polarization and intensity of the associated $\mathrm{BB}$ transitions, i.e., $P_{P L}=\frac{I_{\sigma^{+}}-I_{\sigma^{-}}}{I_{\sigma^{+}}+I_{\sigma^{-}}}$and $I^{\sigma^{+}} / I^{\sigma^{x}}$ (or $I^{\sigma^{-}} / I^{\sigma^{x}}$ ). Here, the superscripts and subscripts represent the polarization of the excitation and emission light, respectively.

Now, we shall experimentally demonstrate that such spin detectors based on the studied GaNAs are, indeed, very effective. In Fig. 2(a), representative PL spectra are shown under circularly polarized (e.g., $\sigma^{+}$, the open circles) and linearly

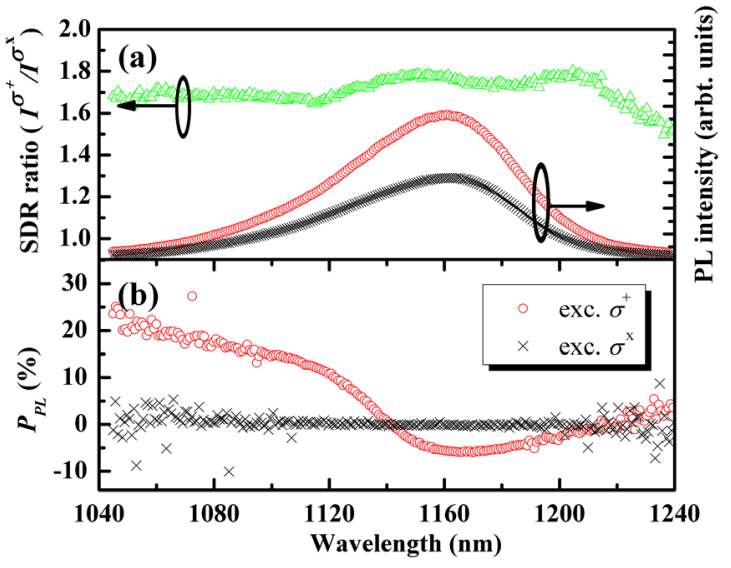

FIG. 2. (Color online) (a) PL, SDR, and (b) polarization spectra obtained at $\mathrm{RT}$ and $0 \mathrm{~T}$ from the $\mathrm{GaN}_{0.026} \mathrm{As}_{0.974}$ epilayer. In (a), the crosses and open circles represent the PL spectra obtained under $\sigma^{x}$ and $\sigma^{+}$excitation, respectively. The open triangles denote the measured SDR ratios. In (b), the crosses and open circles represent the PL polarization curves obtained under $\sigma^{x}$ and $\sigma^{+}$excitation, respectively. All data were obtained under the excitation photon wavelength of $925 \mathrm{~nm}$.

polarized $\left(\sigma^{x}\right)$ excitation. All data shown in Figs. 2-4 were obtained from a GaNAs epilayer with $2.6 \% \mathrm{~N}$ composition grown at $390{ }^{\circ} \mathrm{C}$, taken as an example. Similar results were obtained from all of the studied samples, quantitatively differing only in the efficiency of the spin detection. The fingerprints of the defect-engineered spin filtering effect are apparent in Fig. 2. First, a significant enhancement (about $70 \%$ ) in the PL intensity of the BB transitions can clearly be observed under $\sigma^{+}$excitation as compared with that under $\sigma^{x}$ excitation, giving rise to an SDR ratio of about 1.7. Secondly, the sizable SDR ratio is accompanied by strong PL polarization, up to $25 \%$, by detecting at short wavelengths under $\sigma^{+}$ excitation [Fig. 2(b)]. The observed spectral variation in $P_{P L}$ arises from the overlap between the $\mathrm{BB}$ transitions from the $\mathrm{CB}$ to the heavy-hole (hh) and light-hole (lh) VB states, which contribute to the overall PL band. ${ }^{12,13}$ The CB-hh and CB-lh $\mathrm{BB}$ transitions are known to exhibit opposite optical polarization, leading to co-polarization with the excitation light for the former and counter-polarization for the latter. ${ }^{6}$ The spectral variation in $P_{P L}$ is caused by a hh-lh splitting due to a tensile in-plane strain field in GaNAs, leaving the CB-hh BB at the higher energy (or shorter wavelength) than the CB-lh BB. ${ }^{12,13}$ In contrast, no PL polarization can be detected under $\sigma^{x}$ excitation, as shown in Fig. 2(b), as expected.

To illustrate the significant enhancement of the spin detection efficiency by the spin filtering effect, we examined the spin detector signals (i.e., the PL polarization and the SDR ratio) in a transverse magnetic field that can completely suppress the defect-mediated spin filtering effect. This can be done because the field-induced Larmor precession of the electrons bound to the spin-filtering defects will lead to spin depolarization and will consequently deactivate the spin filtering/blockade effect via these defects. As a result, strong quenching of the PL polarization and the SDR ratio in an applied transverse field, known as a Hanle curve, should be observed. The results are shown in Fig. 3(a) and 3(b) for $I^{\sigma^{+}} / I^{\sigma^{x}}$ and $P_{P L}$, respectively. As can be seen, the spin polarization of the defect electrons can be destroyed in a relative 


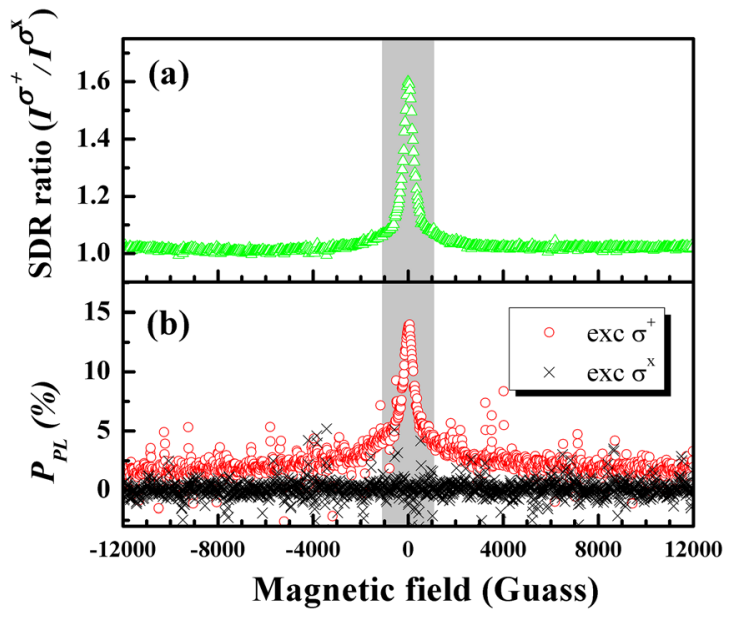

FIG. 3. (Color online) Hanle curves from the $\mathrm{GaN}_{0.026} \mathrm{As}_{0.974}$ epilayer, obtained at RT in a transverse magnetic field by detecting (a) the SDR ratio, and (b) the PL polarization. In (b), the crosses and open circles represent the PL polarization curves obtained under $\sigma^{x}$ and $\sigma^{+}$excitation, respectively. The excitation and detection wavelengths are 925 and $1100 \mathrm{~nm}$, respectively. For easy viewing, the field range where the spin filtering effect is active is marked by the shaded area.

low field $(<0.1 \mathrm{~T})$. An electron spin relaxation time of $\sim 700$ ps, deduced from an analysis of the Hanle curves under the limit of low excitation density, ${ }^{14}$ is noticeably longer than the spin lifetime ( $150 \mathrm{ps})$ of the $\mathrm{CB}$ electrons, ${ }^{9,15}$ making the spin filtering effect effective. We should note that when the spin filtering effect becomes inactive in a high field, the SDR ratio returns to unity and the PL polarization decreases down to only about $2 \%$, even though the initial spin polarization of the photo-generated $\mathrm{CB}$ electrons remains unchanged. This is the case when the spin polarization of the $\mathrm{CB}$ electrons is limited by their own fast spin relaxation and the CB electrons are no longer spin blockaded from being annihilated with holes via the spin-unpolarized defects. Under such conditions, the spin detector becomes too inefficient to be reliable or functional, just as in the cases for most semiconductors without the defect-engineered spin filtering effect. Therefore, the results from the Hanle effect shown in Fig. 3 provide compelling evidence for the drastically increased spin detection efficiency by the spin-filtering effect.

The availability of the highly efficient RT spin detector based on GaNAs, facilitated by the spin filtering effect, has paved the way to assess RT spin injection in related material systems that was not previously possible. As an example, we carried out a study of optical spin injection from a GaAs layer into an adjacent GaNAs spin detector at RT. As shown in Fig. 4, the spin injection from GaAs gives rise to an SDR ratio of about 1.25 . This value is markedly lower than the 1.75 observed under $\sigma^{+}$excitation below the bandgap of GaAs when electron spins are only generated within the spin detector. The dependence of the SDR ratio as a function of excitation wavelength, displayed in the inset of Fig. 4, clearly shows a sharp drop of the SDR ratio when the excitation is tuned above the bandgap of GaAs (i.e., shorter than $870 \mathrm{~nm}$ ). This finding indicates a significant spin loss during the spin injection across the GaAs/GaNAs interface.

In summary, we have shown that the defect-engineered spin filtering/blockade effect can drastically enhance the spin

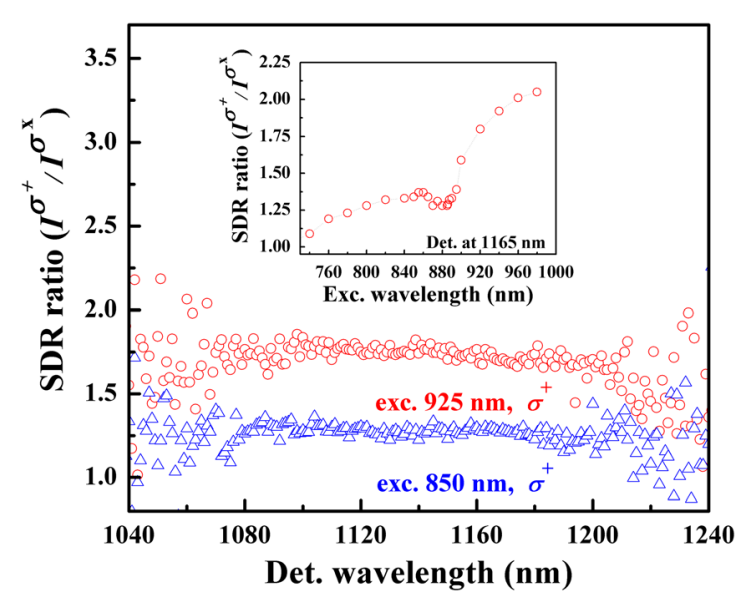

FIG. 4. (Color online) SRD ratio obtained at $\mathrm{RT}$ and $0 \mathrm{~T}$ from the $\mathrm{GaN}_{0.026} \mathrm{As}_{0.974}$ epilayer, under the $\sigma^{+}$excitation at $850 \mathrm{~nm}$ (open triangles) and $925 \mathrm{~nm}$ (open circles). The inset shows the SDR ratio as a function of the excitation wavelengths by detecting at $1165 \mathrm{~nm}$.

polarization of $\mathrm{CB}$ electrons and can turn GaNAs into an efficient spin detector operational at RT. Both the PL polarization and the PL intensity can be taken as spin detection signals, which are sensitive to the initial spin polarization of CB electrons (e.g., spin polarization of injected electrons). We should point out that applications of such spin detectors can be extended beyond optical detection. Since the density of the $\mathrm{CB}$ electrons is also significantly enhanced via the spin filtering/blockade effect, which is evident from the observed SDR ratio $I^{\sigma^{+}} / I^{\sigma^{x}}>1$, GaNAs can also be employed as an electrical spin detector where the spin polarization of incoming $\mathrm{CB}$ electrons is reflected by the electric current density.

${ }^{1}$ S. A. Wolf, D. D. Awschalom, R. A. Buhrman, J. M. Daughton, S. von Molnár, M. L. Roukes, A. Y. Chtchelkanova, and D. M. Treger, Science 294, 1488 (2001).

${ }^{2}$ I. Žutić, J. Fabian, and S. Das Sarma, Rev. Mod. Phys. 76, 323 (2004).

${ }^{3}$ D. D. Awaschalom and M. F. Flatté, Nat. Phys. 3, 153 (2007).

${ }^{4}$ Spintronics, Semiconductors and Semimetals, edited by T. Dietl, D. D. Awschalom, M. Kaminska, and H. Ohno (Academic, New York, 2008), Vol. 82.

${ }^{5}$ Handbook of Spintronic Semiconductors, edited by W. M. Chen and I. A. Buyanova (Pan Stanford, Singapore, 2010).

${ }^{6}$ Optical Orientation, edited by F. Meier and B. P. Zakharchenya (NorthHolland, Amsterdam, 1984).

${ }^{7}$ X. J. Wang, I. A. Buyanova, F. Zhao, D. Lagarde, A. Balocchi, X. Marie, C. W. Tu, J. C. Harmand, and W. M. Chen, Nature Mater. 8, 198 (2009).

${ }^{8}$ V. K. Kalevich, E. L. Ivchenko, M. M. Afanasiev, A. Yu. Shiryaev, A. Yu. Egorov, V. M. Ustinov, and Y. Masumoto, JETP Lett. 82, 455 (2005).

${ }^{9}$ D. Lagarde, L. Lombez, X. Marie, A. Balocchi, T. Amand, V. K. Kalevich, A. Shiryaev, E. Ivechenko, and A. Egorov, Phys. Status Solidi A 204, 208 (2007).

${ }^{10}$ Y. Puttisong, X. J. Wang, I. A. Buyanova, H. Carrére, F. Zhao, A. Balocchi, X. Marie, C. W. Tu, and W. M. Chen, Appl. Phys. Lett. 96, 052104 (2010).

${ }^{11}$ Y. Puttisong, X. J. Wang, I. A. Buyanova, C. W. Tu, L. Geelhaar, H. Riechert, and W. M. Chen, Appl. Phys. Lett. 98, 012112 (2011).

${ }^{12}$ I. A. Buyanova, W. M. Chen, and B. Monemar, MRS Internet J. Nitride Semicond. Res. 6, 2 (2001).

${ }^{13}$ For a review, see Physics and Applications of Dilute Nitrides, edited by I. A. Buyanova and W. M. Chen (Taylor \& Francis, New York, 2004); Dilute III-V Nitride Semiconductors and Material Systems, Springer Series in Material Science Vol.105, edited by A. Erol (Springer, Berlin, 2008).

${ }^{14}$ V. K. Kalevich, A. Yu. Shiryaev, E. L. Ivchenko, M. M. Afanasiev, A. Yu. Egorov, V. M. Ustinov, and Y. Masumoto, Physica B 404, 4929 (2009).

${ }^{15}$ V. K. Kalevich, A. Yu. Shiryaev, E. L. Ivchenko, A. Yu. Egorov, L. Lombez, D. Lagarde, X. Marie, and T. Amand, JETP Lett. 85, 174 (2007). 